\title{
A General Synthesis of Vinylic Silyl Hydrides Using Nickel Catalysis. Applications to the Syntheses of Silylene-Tethered Conjugated Polymers
}

\author{
Ruey-Min Chen and Tien-Yau Luh* \\ Department of Chemistry, National Taiwan University, Taipei, Taiwan 106, Republic of China
}

Received 15 July 1997; accepted 10 September 1997

\begin{abstract}
Treatment of benzylic or allylic dithioacetals with $\mathrm{Me}_{2}\left({ }^{\mathrm{i}} \mathrm{PrO}\right) \mathrm{SiCH}_{2} \mathrm{MgCl}$ in the presence of $\mathrm{NiCl}_{2}\left(\mathrm{PPh}_{3}\right)_{2}$ catalyst yielded the corresponding i-propoxy(vinyl)silanes which are reduced with $\mathrm{LiAlH}_{4}$ to afford vinylic silyl hydrides. These hydrides serve as the precursors for the syntheses of silylene-tethered $\sigma, \pi$-conjugated copolymers. 1998 Elsevier Science Ltd. All rights reserved.
\end{abstract}

\section{Introduction}

Conjugated polymers can display diverse electroactive properties such as photosensitivity, ${ }^{\prime}$ conductivity, ${ }^{2}$ as well as non-linear optical properties. ${ }^{3}$ A polyphenylene-vinylene-based device has been shown to serve as a light emitting diode in the green light region. ${ }^{4}$ Various model systems suggest that the photophysical properties of certain conjugated polymers can be represented by those of a short fragment of the corresponding chromophores. ${ }^{5}$ Accordingly, there has been an increasing study on the use of spacers between well-defined conjugated moieties in the polymeric backbone that can increase the processibility and, in the meantime, the emission wavelength can be predicted. ${ }^{6,7}$ Silylene moiety appears to be an attractive spacer for this purpose. 7 These polymers can be conveniently obtained by a displacement of silicon-halide bond with alkynyl nucleophile. ${ }^{2 b, 6}$ However, the structural variety of such polymers is somewhat limited. More recently, silylene-tethered oligophenylene-vinylene lumiphores 1 were synthesized by Wittig reaction (eq 1). ${ }^{7}$ In these

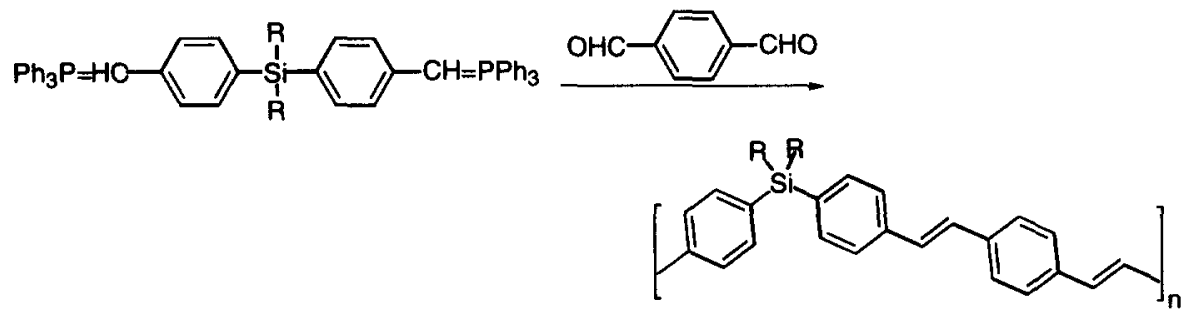

1

studies, the silylene group in $\mathbf{1}$ is linked to two aryl moieties of the backbone such that the steric environment around the silicon atom will be somewhat congested. A replacement of the aryl linkage in 1 by the vinylic ligand may release the steric hindrance at silicon. Hydrosilylation turns out to be the most promising procedure for the synthesis of polyvinylene-silylenes. ${ }^{8-10}$ To illustrate this, platinum-catalyzed polymerization of alkynylsilanes has been used to synthesize 2 (eq 2)..$^{9}$ More recently, reactions of arylene-spaced bisalkynylsilyl hydrides with bisalkynes under similar conditions can furnish the preparation of polymer 3 (eq 
3). ${ }^{10}$ However, these procedures are of limited success because only alkynyl silyl hydride reagents have been employed.

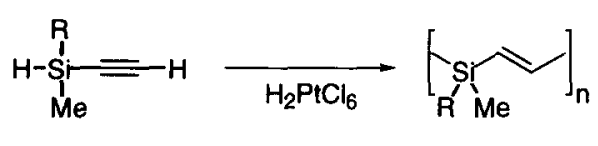

2

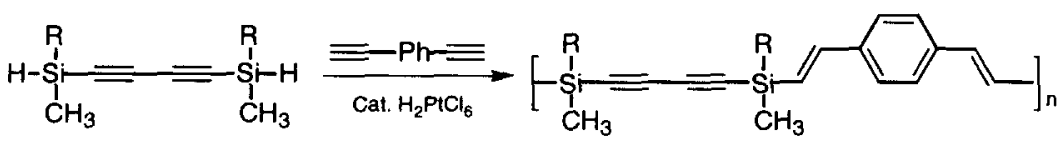

Recently, we were interested in investigating the photophysics of silylene tethered conjugated polymers 6. 11 Our synthetic strategy relies on the hydrosilylation of bisalkynes 4 with vinylic bis-silyl hydrides 5 (eq 4 ). Accordingly, a variety of vinyl silyl hydrides is required for this purpose. To the best of our knowledge, the use of these hydrides in the transition metal-catalyzed hydrosilylation for the synthesis of silylene-tethered polymers has not been reported. It is interesting to note that not many vinylic silyl hydrides are known in the literature. ${ }^{12}$ Chan et al. briefly reported the preparation of ( $\beta$-styryl)dimethylsilane ( 7 ) from the reaction of $\beta$ styryl lithium with dimethylchlorosilane (eq 5). 12a However, these procedures may not be easily applicable for the synthesis of 5 with different structural variety.

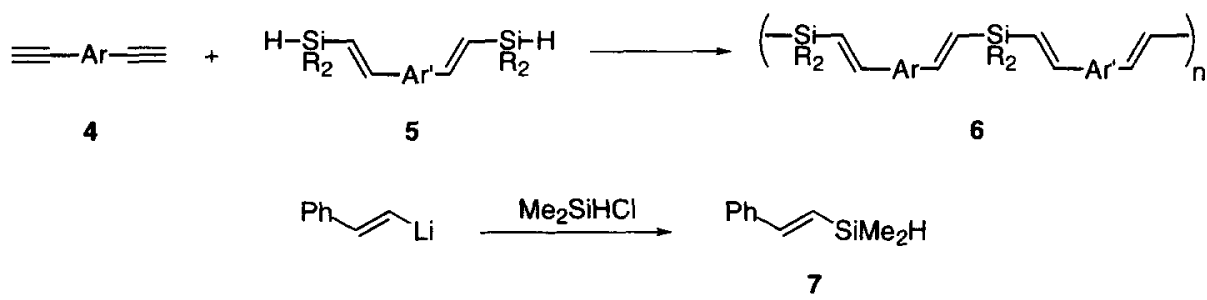

We recently reported a unified procedure to prepare vinylsilanes 9 from the corresponding aryl- or vinylsubstituted dithioacetals 8 (eq 6). ${ }^{13,14}$ Bisvinylsilanes 10 can be prepared conveniently from 11. It was felt that, when appropriate Grignard reagents are employed, this methodology can provide a convenient procedure for the preparation of various vinylic hydrosilane derivatives 12.

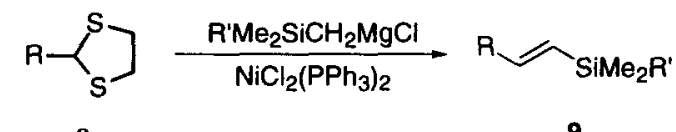

8

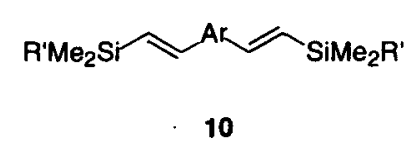

10

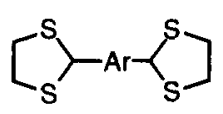

11<smiles>[R]C=C[AsH2]</smiles>

12 


\section{Results and Discussion}

At the beginning of this research, $\mathrm{HMe}_{2} \mathrm{SiCH}_{2} \mathrm{MgCl}^{15}$ was used to attempt the cross coupling reaction with dithioacetal 8 under the nickel-catalyzed conditions. Unfortunately, a mixture of products was obtained and the corresponding vinylic silyl hydride 12 was isolated, at most, as the minor product. An alternative route is therefore highly desirable.

It is well documented that silyl hydrides can be synthesized by reduction of the corresponding silyl halides ${ }^{16}$ or triflates. ${ }^{17}$ More recently, treatment of alkoxysilane moiety in polymers by $\mathrm{LiAlH}_{4}$ also led to the formation of silyl hydride functionality. ${ }^{18}$ Accordingly, it is envisaged that this methodology can be extended to the synthesis of 12 . Our strategy is therefore to adopt the reaction shown in eq 6, using $\mathrm{Me}_{2}(\mathrm{iPrO}) \mathrm{SiCH}_{2} \mathrm{MgCl}$ as the Grignard reagent. ${ }^{19}$ The corresponding isopropoxyvinylsilanes 13 would be expected. Reduction of the Si-O bond in $\mathbf{1 3}$ would lead to the formation of the corresponding vinylic silyl hydrides 12.

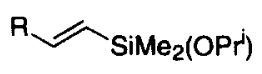

13
$(\mathrm{PrO}) \mathrm{Me}_{2} \mathrm{Si} \approx \mathrm{AR} / \mathrm{SiMe}_{2}(\mathrm{OPr})$

14

A benzene solution of dithioacetal 8 and $\mathrm{Me}_{2}\left({ }^{(} \mathrm{PrO}\right) \mathrm{SiCH}_{2} \mathrm{MgCl}$ in the presence of $5 \mathrm{~mol} \%$ of $\mathrm{NiCl}_{2}\left(\mathrm{PPh}_{3}\right)_{2}$ was refluxed for $16 \mathrm{~h}$ to give, after usual workup, the corresponding vinylsilane 13 in good yield. Reactions of bisdithioacetals 11 under the same conditions yielded bisvinylsilanes 14. The results are tabulated in Table 1. It is noteworthy that aromatic hydrocarbon solvents were essential for these cross coupling

Table $1 \mathrm{NiCl}_{2}\left(\mathrm{PPh}_{3}\right)_{2}$-Catalyzed Cross Coupling Reactions of 8 or 11 with $\mathrm{Me}_{2}$ ('PrO) $\mathrm{SiCH}_{2} \mathrm{MgCl}$

\begin{tabular}{|c|c|c|}
\hline Entry & Substrate (8 or 11) & Product (\% Yield) \\
\hline 1 & $8 \mathbf{a}(\mathrm{R}=\mathrm{Ph})$ & $13 a(85)$ \\
\hline 2 & $8 \mathrm{~b}(\mathrm{R}=\mathrm{2}-\mathrm{Thieny})$ & $13 \mathrm{~b}(90)$ \\
\hline 3 & $8 c(R=\beta$-styryl) & $13 c(88)$ \\
\hline 4 & $11 \mathbf{a}(\mathrm{Ar}=$ & $14 a(58)$ \\
\hline 5 & $11 \mathrm{~b}(\mathrm{Ar}=$ & $14 b(56)$ \\
\hline 6 & $11 \mathrm{c}(\mathrm{Ar}=$ & $14 c(84)$ \\
\hline 7 & $11 d(A r=$ & $14 d(53)$ \\
\hline
\end{tabular}


reactions when benzylic substrates $\mathbf{8 a}, \mathbf{b}$, and 11 were employed (entries 1,2,4-7). However, allylic dithioacetal $8 \mathrm{c}$ can undergo the reaction without difficulty in THF solvent (entry 3 ).

Reduction of the $\mathrm{Si}-\mathrm{O}$ bond in 13 and 14 with $\mathrm{LiAlH}_{4}$ in refluxing benzene solution for $16-18 \mathrm{~h}$ yielded the corresponding silylhydrides 12 and 5 , respectively. Longer reaction time was necessary when the reaction was carried out at lower temperature (e.g. in refluxing ether). The results are summarized in Table 2.

Table 2 Reduction of 13 or 14 with $\mathrm{LiAlH}_{4}$

\begin{tabular}{|c|c|c|}
\hline Entry & Substrate (13 or 14$)$ & Product (\% Yield) \\
\hline 8 & $13 \mathbf{a}(\mathrm{R}=\mathrm{Ph})$ & $12 a(75)$ \\
\hline 9 & 13b $(\mathrm{R}=2-$ Thienyl) & $12 b(74)$ \\
\hline 10 & $13 c(R=\beta$-styryl) & $12 c(65)$ \\
\hline 11 & $14 \mathbf{a}(\mathrm{Ar}=$ & $5 \mathbf{a}(75)$ \\
\hline 12 & $14 b(\mathrm{Ar}=$ & $5 b(74)$ \\
\hline 13 & $14 c(\mathrm{Ar}=$ & $5 c(73)$ \\
\hline 14 & $14 d(A r=$ & $5 d(67)$ \\
\hline
\end{tabular}

As evident in Table 2, vinyl silyl hydrides 5 and 12 can be conveniently prepared in two steps in satisfactory yield. Since the aryl group in $\mathbf{8}$ and $\mathbf{1 1}$ can be easily modified, a wide range of different structural variety of vinylic silyl hydrides $\mathbf{5}$ and $\mathbf{1 2}$ can thus be synthesized. It is noteworthy that these hydrides $\mathbf{5}$ have served as a useful entity for the synthesis of silylene-tethered conjugated oligomers $\mathbf{1 5}$ and polymers 6.11 To illustrate this, phenylacetylene was allowed to react with $\mathbf{5 b}$ in the presence of $0.5 \mathrm{~mol} \%$ of $\mathrm{RhCl}\left(\mathrm{PPh}_{3}\right)_{3}$ to afford 15a (eq 7). Oligomer 15b was prepared in a similar manner. Alternatively, 12b was employed to add onto 1,4-diethynylbenzene to yield 15c (eq 8). When bis-alkynes 4 and bis-silyl hydrides 5 were allowed to react under similar conditions, the silylene tethered $\sigma-\pi$ conjugated polymers 6 were thus obtained (eq 9 ).

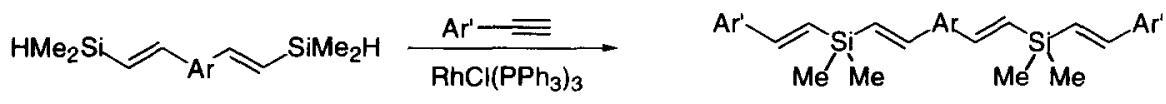

$5 b$ or $5 c$

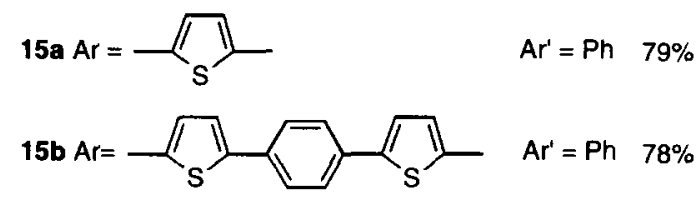



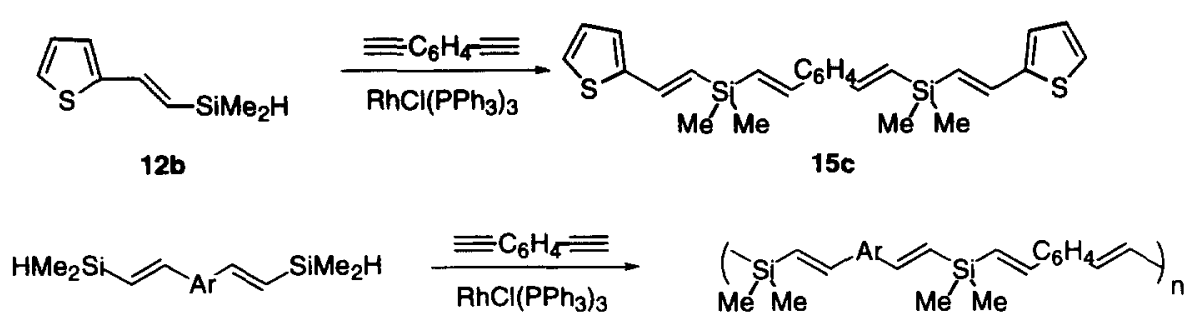

$5 b$ or $5 c$

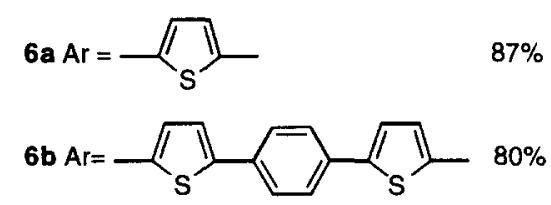

In summary, we have demonstrated a convenient two-step synthesis of a variety of vinylic silyl hydrides from the corresponding dithioacetals. These hydrides can be extended to the synthesis of a wide range of silylene-tethered conjugated oligomers and polymers. The investigation of the photophysical properties of these oligomers as well as polymers is in progress in our laboratory.

\section{Experimental Section}

General Procedure for the Preparation of Bisdithioacetals 11. - A chloroform solution of the dialdehyde ( 1 equiv), $\mathrm{BF}_{3}{ }^{\bullet} \mathrm{Et}_{2} \mathrm{O}(10 \mathrm{~mol} \%$ ) and 1,2-ethanedithiol (3.0 equiv) was stirred at room temperature for $4 \mathrm{~h}$. The mixture was poured into $10 \% \mathrm{NaOH}$ and the two layers were separated. The organic layer was washed with brine $(20 \mathrm{~mL})$, and dried $\left(\mathrm{MgSO}_{4}\right)$. The solvent was removed in vacuo to give 11 .

$11 \mathrm{~b}(99 \%) ; \mathrm{mp} 68-70^{\circ} \mathrm{C}$; ${ }^{1} \mathrm{H} \mathrm{NMR}\left(\mathrm{CDCl}_{3}, 300 \mathrm{MHz}\right) \delta 3.26-3.47(\mathrm{~m}, 8 \mathrm{H}), 5.79(\mathrm{~s}, 2 \mathrm{H}), 6.80$ (s, 2 $\mathrm{H}) ;{ }^{13} \mathrm{C} \mathrm{NMR}\left(\mathrm{CDCl}_{3}, 75 \mathrm{MHz}\right) \delta 39.9,50.9,124.9,147.2 ; \mathrm{MS} \mathrm{m} / \mathrm{z}$ (rel intensity) $292\left(\mathrm{M}^{+}, 100\right), 264(41)$, 172 (28), 127 (26), 105 (12), 59 (7); HRMS Calcd for $\mathrm{C}_{10} \mathrm{H}_{12} \mathrm{~S}_{5}$ : 291.9542 ; Found: 291.9537.

$11 \mathrm{c}(92 \%)$; mp 195-197\% $\mathrm{C}$; 'H NMR $\left(\mathrm{CDCl}_{3}, 300 \mathrm{MHz}\right) \delta 3.35-3.52(\mathrm{~m}, 8 \mathrm{H}), 5.89(\mathrm{~s}, 2 \mathrm{H}), 7.00(\mathrm{~d}$, $J=3.7 \mathrm{~Hz}, 2 \mathrm{H}), 7.08(\mathrm{~d}, J=3.7 \mathrm{~Hz}, 2 \mathrm{H}), 7.52(\mathrm{~s}, 4 \mathrm{H}) ;{ }^{13} \mathrm{C} \mathrm{NMR}\left(\mathrm{CDCl}_{3}, 75 \mathrm{MHz}\right) \delta 39.9,50.9,122.5$, $125.9,126.0,126.5,143.8,146.6$; MS m/z (rel intensity) $450\left(\mathrm{M}^{+}, 100\right), 422$ (43), 361 (19), 328 (35), 285 (14), 242 (121), 196 (7), 183 (7), 124 (26); HRMS Calcd for $\mathrm{C}_{20} \mathrm{H}_{18} \mathrm{~S}_{6}$ : 449.9733; Found: 449.9728.

$11 \mathrm{~d}(68 \%):{ }^{1} \mathrm{H}$ NMR $\left(\mathrm{CDCl}_{3}, 300 \mathrm{MHz}\right) \delta 3.31-3.52(\mathrm{~m}, 8 \mathrm{H}), 5.89(\mathrm{~s}, 2 \mathrm{H}), 6.91(\mathrm{~d}, J=3.6 \mathrm{~Hz}, 2$ H), $6.93(\mathrm{~d}, J=3.6 \mathrm{~Hz}, 2 \mathrm{H}), 6.99(\mathrm{~s}, 2 \mathrm{H}) ;{ }^{13} \mathrm{C} \mathrm{NMR}\left(\mathrm{CDCl}_{3}, 50 \mathrm{MHz}\right) \delta 39.9,50.9,123.0,124.2,126.2$, 136.2, 137.2, 146.5; MS m/z (rel intensity) $456\left(\mathrm{M}^{+}, 14\right), 369$ (15), 380 (13), 277 (100), 264 (10), 184 (18), 124 (53), 64 (30); HRMS Calcd for $\mathrm{C}_{18} \mathrm{H}_{16} \mathrm{~S}_{7}$ : 455.9297 ; Found: 455.9292.

General Procedure for the $\mathrm{NiCl}_{2}\left(\mathrm{PPh}_{3}\right)_{2}$-Catalyzed Reactions of Dithioacetals 8 or 11 with $\mathrm{Me}_{2}$ ( $\left.{ }^{\mathrm{PPrO}}\right) \mathrm{SiCH}_{2} \mathrm{MgCl}$. A THF solution of $\mathrm{Me}_{2}\left({ }^{(} \mathrm{PrO}\right) \mathrm{SiCH}_{2} \mathrm{MgCl}$ (5-10 equiv) was evacuated as much as possible and then dissolved in benzene. Dithioacetal 8 or 11 and $\mathrm{NiCl}_{2}\left(\mathrm{PPh}_{3}\right)_{2}(0.1$ equiv) were then introduced. The mixture was refluxed under $\mathrm{N}_{2}$ for $16 \mathrm{~h}$ and then poured into saturated $\mathrm{NH}_{4} \mathrm{Cl}$. The organic layer was separated and the aqueous layer was extracted twice $(2 \times 20 \mathrm{~mL})$ with ether. The combined organic portions were washed twice $(2 \times 20 \mathrm{~mL})$ with $10 \% \mathrm{NaOH}$ and with brine $(20 \mathrm{~mL})$, and dried $\left(\mathrm{MgSO}_{4}\right)$. The 
solvent was removed in vacuo and the residue was distilled under reduced pressure or triturated with $\mathrm{MeOH}$ to give compound 13 or 14.

Dimethyl(i-propoxy)( $\beta$-styryl)silane (13a). In a manner similar to that described in the general procedure, a mixture of $8 \mathrm{a}(1.82 \mathrm{~g}, 10.0 \mathrm{mmol})$ and $\mathrm{NiCl}_{2}\left(\mathrm{PPh}_{3}\right)_{2}(0.70 \mathrm{~g}, 1.0 \mathrm{mmol})$ was treated with $\mathrm{Me}_{2}\left({ }^{(} \mathrm{PrO}\right) \mathrm{SiCH}_{2} \mathrm{MgCl}(50.0 \mathrm{mmol})$ in benzene $(50 \mathrm{~mL})$ to give $13 \mathrm{a}(1.55 \mathrm{~g}, 85 \%)$ : bp $64{ }^{\circ} \mathrm{C}(0.5 \mathrm{~mm}){ }^{1} \mathrm{H}$ NMR $\left(\mathrm{CDCl}_{3}, 200 \mathrm{MHz}\right) \delta 0.26(\mathrm{~s}, 6 \mathrm{H}), 1.17(\mathrm{~d}, J=5.7 \mathrm{~Hz}, 6 \mathrm{H}), 4.03$ (sept, $\left.J=5.7 \mathrm{~Hz}, 1 \mathrm{H}\right), 6.43(\mathrm{~d}, J$ $=19.3 \mathrm{~Hz}, 1 \mathrm{H}), 6.97(\mathrm{~d}, J=19.3 \mathrm{~Hz}, 1 \mathrm{H}), 7.27-7.46(\mathrm{~m}, 5 \mathrm{H}) ;{ }^{13} \mathrm{C} \mathrm{NMR}\left(\mathrm{CDCl}_{3}, 50 \mathrm{MHz}\right) \delta-1.1,25.7$, $65.1,126.5,127.1,128.3,128.5,138.0,145.2 ; \mathrm{MS} \mathrm{m} / \mathrm{z}$ (rel intensity) $220\left(\mathrm{M}^{+}, 34\right), 189(86), 162$ (68), 147 (100), 131 (42), 117 (40), 75 (42), 59 (14); HRMS Calcd for $\mathrm{C}_{13} \mathrm{H}_{20} \mathrm{SiO}: 220.1283$; Found: 220.1290.

Dimethyl(i-propoxy)( $\beta$-thienylvinyl)silane (13b). In a manner similar to that described in the general procedure, a mixture of $8 \mathbf{b}(1.88 \mathrm{~g}, 10.0 \mathrm{mmol}), \mathrm{NiCl}_{2}\left(\mathrm{PPh}_{3}\right)_{2}(0.70 \mathrm{~g}, 1.0 \mathrm{mmol})$ and $\mathrm{Me}_{2}\left({ }^{(} \mathrm{PrO}\right) \mathrm{SiCH}_{2} \mathrm{MgCl}(50.0 \mathrm{mmol})$ in benzene $(50 \mathrm{~mL})$ was transformed into $13 \mathrm{~b}(1.69 \mathrm{~g}, 90 \%)$ : bp $62{ }^{\circ} \mathrm{C}$ $(0.5 \mathrm{~mm}){ }^{1} \mathrm{H}$ NMR $\left(\mathrm{CDCl}_{3}, 200 \mathrm{MHz}\right) \delta 0.24(\mathrm{~s}, 6 \mathrm{H}), 1.16(\mathrm{~d}, J=6.1 \mathrm{~Hz}, 6 \mathrm{H}), 4.03$ (sept, $J=6.1 \mathrm{~Hz}, 1$ H), $6.17(\mathrm{~d}, J=18.9 \mathrm{~Hz}, 1 \mathrm{H}), 6.94-7.09(\mathrm{~m}, 3 \mathrm{H}), 7.18(\mathrm{~d}, J=4.7 \mathrm{~Hz}, 1 \mathrm{H}) ;{ }^{13} \mathrm{C} \mathrm{NMR}\left(\mathrm{CDCl}_{3}, 50 \mathrm{MHz}\right)$ $\delta-1.2,25.7,65.1,125.1,126.2,126.6,127.4,137.6,144.9 ; \mathrm{MS} \mathrm{m} / \mathrm{z}$ (rel intensity) $226\left(\mathrm{M}^{+}, 15\right), 211$ (7), 189 (7), 168 (100), 151 (66), 143 (32), 133 (20), 75 (36), 59 (17); HRMS Calcd for $\mathrm{C}_{11} \mathrm{H}_{18} \mathrm{SiOS}: 226.0847$; Found: 226.0855 .

1,4-Bis \{[dimethyl(i-propoxy)silyl] vinyl\}benzene (14a). In a manner similar to that described in the general procedure, a mixture of $11 \mathrm{a}(1.43 \mathrm{~g}, 5.0 \mathrm{mmol})$ and $\mathrm{NiCl}_{2}\left(\mathrm{PPh}_{3}\right)_{2}(0.35 \mathrm{~g}, 0.5 \mathrm{mmol})$ was treated with $\mathrm{Me}_{2}\left({ }^{(P r O}\right) \mathrm{SiCH}_{2} \mathrm{MgCl}(50.0 \mathrm{mmol})$ in benzene $(50 \mathrm{~mL})$ was converted to $14 \mathrm{a}(1.05 \mathrm{~g}, 58 \%) ; \mathrm{bp}$ $88{ }^{\circ} \mathrm{C}(0.5 \mathrm{~mm}){ }^{1} \mathrm{H}$ NMR $\left(\mathrm{CDCl}_{3}, 300 \mathrm{MHz}\right) \delta 0.25(\mathrm{~s}, 12 \mathrm{H}), 1.17(\mathrm{~d}, J=5.8 \mathrm{~Hz}, 12 \mathrm{H}), 4.03(\mathrm{sept}, J=$ $5.8 \mathrm{~Hz}, 2 \mathrm{H}), 6.43(\mathrm{~d}, J=19.2 \mathrm{~Hz}, 2 \mathrm{H}), 6.95(\mathrm{~d}, J=19.2 \mathrm{~Hz}, 2 \mathrm{H}), 7.41(\mathrm{~s}, 4 \mathrm{H}) ;{ }^{13} \mathrm{C} \mathrm{NMR}\left(\mathrm{CDCl}_{3}, 50\right.$ MHz): $\delta-1.1,25.7,65.1,126.8,133.5,133.9,144.7 ; \mathrm{MS} \mathrm{m} / \mathrm{z}$ (rel intensity) $362\left(\mathrm{M}^{+}, 34\right), 347$ (14), 304 (100), 261 (78), 219 (11), 189 (38), 147 (19), 133 (26), 117 (58), 75 (19); HRMS Calcd for $\mathrm{C}_{20} \mathrm{H}_{34} \mathrm{Si}_{2} \mathrm{O}_{2}$ 362.2097; Found: 362.2103 .

2,5-Bis $\{\boldsymbol{\beta}$-[dimethyl(i-propoxy)silyl]vinyl $\}$ thiophene (14b). In a manner similar to that described in the general procedure, a mixture of $11 \mathrm{~b}(1.46 \mathrm{~g}, 5.0 \mathrm{mmol})$ and $\mathrm{NiCl}_{2}\left(\mathrm{PPh}_{3}\right)_{2}(0.35 \mathrm{~g}, 0.5 \mathrm{mmol})$ was treated with $\mathrm{Me}_{2}\left({ }^{\mathrm{PrO}}\right) \mathrm{SiCH}_{2} \mathrm{MgCl}(50.0 \mathrm{mmol})$ in benzene $(50 \mathrm{~mL})$ to give $14 \mathrm{~b}(1.03 \mathrm{~g}, 56 \%)$; bp $92{ }^{\circ} \mathrm{C}$ $(0.5 \mathrm{~mm})^{1} \mathrm{H} \mathrm{NMR}\left(\mathrm{CDCl}_{3}, 200 \mathrm{MHz}\right) \delta 0.23(\mathrm{~s}, 12 \mathrm{H}), 1.17(\mathrm{~d}, J=5.9 \mathrm{~Hz}, 12 \mathrm{H}), 4.99$ (sept, $J=5.9 \mathrm{~Hz}$, $2 \mathrm{H}), 6.15(\mathrm{~d}, J=18.8 \mathrm{~Hz}, 2 \mathrm{H}), 6.85(\mathrm{~s}, 2 \mathrm{H}), 6.96(\mathrm{~d}, J=18.8 \mathrm{~Hz}, 2 \mathrm{H}) ;{ }^{13} \mathrm{C} \mathrm{NMR}\left(\mathrm{CDCl}_{3}, 75 \mathrm{MHz}\right) \delta$ $-1.2,25.7,65.2,126.9,127.5,137.7,144.5 ; \mathrm{MS} \mathrm{m} / \mathrm{z}$ (rel intensity) $368\left(\mathrm{M}^{+}, 45\right), 354(10), 278(100), 189$ (41), 147 (15), 108 (32), 75 (55); HRMS Calcd for $\mathrm{C}_{18} \mathrm{H}_{32} \mathrm{Si}_{2} \mathrm{O}_{2} \mathrm{~S}: 368.1661$ Found: 368.1664.

2,2'-p-Phenylene-bis-5-[ $\beta$-dimethyl(i-propoxy)silylvinyl]thiophene $(\mathbf{1 4 c})$. In a manner similar to that described in the general procedure, a mixture of $11 \mathrm{c}(2.25 \mathrm{~g}, 5.0 \mathrm{mmol}), \mathrm{NiCl}_{2}\left(\mathrm{PPh}_{3}\right)_{2}(0.35 \mathrm{~g}$, $0.5 \mathrm{mmol})$ and $\mathrm{Me}_{2}(\mathrm{PrO}) \mathrm{SiCH}_{2} \mathrm{MgCl}(50.0 \mathrm{mmol})$ in benzene $(50 \mathrm{~mL})$ was transformed into $14 \mathrm{c}(2.2 \mathrm{~g}$. 84\%); mp 102-103 ${ }^{\circ} \mathrm{C}$; ' ${ }^{\mathrm{H}} \mathrm{NMR}\left(\mathrm{CDCl}_{3}, 300 \mathrm{MHz}\right) \delta 0.25$ (s, $12 \mathrm{H}$ ), $1.17(\mathrm{~d}, J=6.0 \mathrm{~Hz}, 12 \mathrm{H}$ ), 4.03 (sept, $J=6.0 \mathrm{~Hz}, 2 \mathrm{H}), 6.18(\mathrm{~d}, J=18.9 \mathrm{~Hz}, 2 \mathrm{H}), 6.96(\mathrm{~d}, J=3.7 \mathrm{~Hz}, 2 \mathrm{H}), 7.01(\mathrm{~d}, J=18.9 \mathrm{~Hz}, 2 \mathrm{H}), 7.2 \mathrm{I}$ (d, $J=3.7 \mathrm{~Hz}, 2 \mathrm{H}), 7.58(\mathrm{~s}, 4 \mathrm{H}) ;{ }^{13} \mathrm{C} \mathrm{NMR}\left(\mathrm{CDCl}_{3}, 75 \mathrm{MHz}\right) \delta-1.1,25.8,65.2,123.6,126.0,127.0,127.6$, 133.4, 137.6, 143.3,144.4; MS m/z (rel intensity) 526 ( $\left.\mathrm{M}^{+}, 47\right), 468$ (14), 410 (32), 352 (14), 256 (21), 213 (10), 185 (15), 171 (10), 129 (45), 97 (60), 85 (49), 73 (100); HRMS Calcd for $\mathrm{C}_{28} \mathrm{H}_{38} \mathrm{Si}_{2} \mathrm{O}_{2} \mathrm{~S}_{2}$ : 526.1851 ; 
Found: 526.1854 .

$5,5^{\prime \prime}-B i s\left\{[\beta\right.$-dimethyl(i-propoxy)silyl]vinyl $\}-2,2^{\prime} ; 5^{\prime} 2^{\prime \prime}$-terthiophene (14d). In a manner similar to that described in the general procedure, a mixture of $11 \mathrm{~d}(1.82 \mathrm{~g}, 4.0 \mathrm{mmol})$ and $\mathrm{NiCl}_{2}\left(\mathrm{PPh}_{3}\right)_{2}(0.28$ $\mathrm{g}, 0.4 \mathrm{mmol})$ was treated with $\mathrm{Me}_{2}\left({ }^{(} \mathrm{PrO}\right) \mathrm{SiCH}_{2} \mathrm{MgCl}(40.0 \mathrm{mmol})$ in benzene $(40 \mathrm{~mL})$ to give $14 \mathrm{~d}(1.13 \mathrm{~g}$, 53\%); ' $\mathrm{H} \mathrm{NMR}\left(\mathrm{CDCl}_{3}, 200 \mathrm{MHz}\right) \delta 0.24(\mathrm{~s}, 12 \mathrm{H}), 1.16(\mathrm{~d}, J=6.1 \mathrm{~Hz}, 12 \mathrm{H}), 4.03$ (sept, $J=6.1 \mathrm{~Hz}, 2$ H), $6.13(\mathrm{~d}, J=18.8 \mathrm{~Hz}, 2 \mathrm{H}), 6.89(\mathrm{~d}, J=3.7 \mathrm{~Hz}, 2 \mathrm{H}), 6.97(\mathrm{~d}, J=18.8 \mathrm{~Hz}, 2 \mathrm{H}), 7.03(\mathrm{~d}, J=3.7 \mathrm{~Hz}, 2$ H), $7.06(\mathrm{~s}, 2 \mathrm{H}) ;{ }^{13} \mathrm{C} \mathrm{NMR}\left(\mathrm{CDCl}_{3}, 50 \mathrm{MHz}\right) \delta-1.2,25.7,65.2,124.0,124.5,127.2,127.3,136.4$, 136.6, 137.4, 143.9; MS m/z (rel intensity) $532\left(\mathrm{M}^{+}, 10\right), 475$ (5), 430 (22), 355 (8), 277 (65), 207 (100), 147 (38), 171 (10), 73 (30); HRMS Calcd for $\mathrm{C}_{26} \mathrm{H}_{36} \mathrm{Si}_{2} \mathrm{O}_{2} \mathrm{~S}_{3}$ : 532.1416; Found: 532.1420.

General Procedure for the reduction of 13 or 14 with LiAlH $_{4}$. A slurry of 13 or 14 and $\mathrm{LiAlH}_{4}$ (2-4 equiv) was refluxed under $\mathrm{N}_{2}$ for $18 \mathrm{~h}$ and cooled to $\mathrm{rt}$. The mixture was then quenched with water. After filtration, the organic layer was separated, dried and the solvent was removed in vacuo to give the residue which was chromatographed on silica gel (hexane) to afford 12 or 5 .

Dimethyl( $\beta$-styryl)silane (12a). In a manner similar to that described in the general procedure, a mixture of $13 \mathrm{a}(2.20 \mathrm{~g}, 10.0 \mathrm{mmol})$ and $\mathrm{LiAlH}_{4}(0.8 \mathrm{~g}, 20.0 \mathrm{mmol})$ in benzene $(25 \mathrm{~mL})$ was transformed into $12 \mathrm{a}(1.21 \mathrm{~g}, 75 \%):{ }^{1} \mathrm{H} \mathrm{NMR}\left(\mathrm{CDCl}_{3}, 300 \mathrm{MHz}\right) \delta 0.25(\mathrm{~d}, J=3.7 \mathrm{~Hz}, 6 \mathrm{H}), 4.22$ (doublet of septet, $J=3.7$, $2.4 \mathrm{~Hz}, 1 \mathrm{H}), 6.46(\mathrm{dd}, J=19.1,2.4 \mathrm{~Hz}, 1 \mathrm{H}), 6.98(\mathrm{~d}, J=19.1 \mathrm{~Hz}, 1 \mathrm{H}), 7.26-7.36(\mathrm{~m}, 3 \mathrm{H}), 7.44(\mathrm{~d}, J=$ $7.6 \mathrm{~Hz}, 2 \mathrm{H}) ;{ }^{13} \mathrm{C} \mathrm{NMR}\left(\mathrm{CDCl}_{3}, 75 \mathrm{MHz}\right) \delta-4.0,126.1,126.4,128.1,128.5,138.1,145.3 ; \mathrm{MS} \mathrm{m} / \mathrm{z}$ (rel intensity) $162\left(\mathrm{M}^{+}, 100\right), 147(74), 131(65), 117(15), 75(14)$; HRMS Calcd for $\mathrm{C}_{10} \mathrm{H}_{14} \mathrm{Si}: 162.0865$; Found: 162.0848.

Dimethyl[ $\beta$-(2-thienyl)vinyl]silane (12b). In a manner similar to that described in the general procedure, a mixture of $13 \mathrm{~b}(2.26 \mathrm{~g}, 10.0 \mathrm{mmol})$ and $\mathrm{LiAlH}_{4}(0.8 \mathrm{~g}, 20.0 \mathrm{mmol})$ in benzene $(25 \mathrm{~mL})$ was allowed to react to give $12 \mathrm{~b}(1.24 \mathrm{~g}, 74 \%):{ }^{1} \mathrm{H}$ NMR $\left(\mathrm{CDCl}_{3}, 300 \mathrm{MHz}\right) \delta 0.23(\mathrm{~d}, J=3.7 \mathrm{~Hz}, 6 \mathrm{H}), 4.01$ (doublet of septet, $J=3.7,2.3 \mathrm{~Hz}, 1 \mathrm{H}), 6.22(\mathrm{dd}, J=18.7,2.3 \mathrm{~Hz}, 1 \mathrm{H}), 6.96-6.98(\mathrm{~m}, 2 \mathrm{H}), 7.05(\mathrm{~d}, J=$ $18.7 \mathrm{~Hz}, 1 \mathrm{H}), 7.18(\mathrm{~d}, J=4.6 \mathrm{~Hz}, 1 \mathrm{H}) ;{ }^{13} \mathrm{C} \mathrm{NMR}\left(\mathrm{CDCl}_{3}, 75 \mathrm{MHz}\right) \delta-4.1,125.0,125.7,125.8,127.4$, 137.8, 144.9; MS m/z (rel intensity) $168\left(\mathrm{M}^{+}, 48\right), 153(100), 141(1.0), 127(41), 75$ (1.0); HRMS Calcd for $\mathrm{C}_{8} \mathrm{H}_{12} \mathrm{SiS}$ : 168.0429; Found: 168.0433 .

Dimethyl[4-phenyl-(1E,3E)-butadien-1-yl]silane (12c). In a manner similar to that described in the general procedure, a mixture of $13 \mathrm{c}(2.46 \mathrm{~g}, 10.0 \mathrm{mmol})$ was treated with $\mathrm{LiAlH}_{4}(0.8 \mathrm{~g}, 20.0 \mathrm{mmol})$ in benzene $(25 \mathrm{~mL})$ to give $12 \mathrm{c}(0.94 \mathrm{~g}, 65 \%){ }^{\prime} \mathrm{H}$ NMR $\left(\mathrm{CDCl}_{3}, 200 \mathrm{MHz}\right) \delta 0.21(\mathrm{~d}, J=3.5 \mathrm{~Hz}, 6 \mathrm{H}), 4.17$ (doublet of septet, $J=3.5,2.4 \mathrm{~Hz}, 1 \mathrm{H}), 5.99(\mathrm{dd}, J=17.2,2.4 \mathrm{~Hz}, 1 \mathrm{H}), 6.56-6.83(\mathrm{~m}, 3 \mathrm{H}), 7.23--7.44$ $(\mathrm{m}, 5 \mathrm{H}) ;{ }^{13} \mathrm{C} \mathrm{NMR}\left(\mathrm{CDCl}_{3}, 50 \mathrm{MHz}\right) \delta-4.0,126.6,127.8,128.6,131.2,131.3,133.4,137.1,145.8$; $\mathrm{MS}$ $\mathrm{m} / \mathrm{z}$ (rel intensity) $188\left(\mathrm{M}^{+}, 70\right), 173(100), 160(8), 145$ (68), 128 (50), 121 (22), 105 (20); HRMS Calcd for $\mathrm{C}_{12} \mathrm{H}_{16} \mathrm{Si}$ : 188.1021; Found: 188.1038.

1,4-Bis[ $\beta$-(dimethylsilyl)vinyl]benzene (5a). In a manner similar to that described in the general procedure, a mixture of $14 \mathrm{a}(1.81 \mathrm{~g}, 5.0 \mathrm{mmol})$ was treated under $\mathrm{N}_{2}$ with $\mathrm{LiAlH}_{4}(0.8 \mathrm{~g}, 20.0 \mathrm{mmol})$ in benzene ( $25 \mathrm{~mL}$ ) to give $5 \mathrm{a}(0.92 \mathrm{~g}, 75 \%)$ : ${ }^{1} \mathrm{H}$ NMR $\left(\mathrm{CDCl}_{3}, 200 \mathrm{MHz}\right) \delta 0.23(\mathrm{~d}, J=3.8 \mathrm{~Hz}, 12 \mathrm{H}), 4.19$ (doublet of septet, $J=3.8,2.7 \mathrm{~Hz}, 2 \mathrm{H}$ ), $6.45(\mathrm{dd}, J=19.2,2.7 \mathrm{~Hz}, 2 \mathrm{H}$ ), $6.95(\mathrm{~d}, J=19.2 \mathrm{~Hz}, 2 \mathrm{H}$ ), 7.39 (s, $4 \mathrm{H}) ;{ }^{13} \mathrm{C} \mathrm{NMR}\left(\mathrm{CDCl}_{3}, 75 \mathrm{MHz}\right) \delta-4.0,126.3,126.7,137.9,144.9 ; \mathrm{MS} \mathrm{m} / \mathrm{z}$ (rel intensity) $246\left(\mathrm{M}^{+}\right.$, 16), $218(10), 171$ (25), 145 (24), 116 (13), 85 (11), 73 (15), 59 (100); HRMS Calcd for $\mathrm{C}_{14} \mathrm{H}_{22} \mathrm{Si}_{2}$ : 
246.1260; Found: 246.1254 .

2,5-Bis[ $\beta$-(dimethylsilyl)vinyl]thiophene (5b). In a manner similar to that described in the general procedure, a mixture of $14 \mathrm{~b}(2.26 \mathrm{~g}, 10.0 \mathrm{mmol})$ was treated with $\mathrm{LiAlH}_{4}(1.6 \mathrm{~g}, 40.0 \mathrm{mmol})$ in benzene $(50 \mathrm{~mL})$ to give $5 \mathrm{~b}(1.24 \mathrm{~g}, 74 \%)$ : ${ }^{1} \mathrm{H} \mathrm{NMR}\left(\mathrm{CDCl}_{3}, 300 \mathrm{MHz}\right) \delta 0.23(\mathrm{~d}, J=3.7 \mathrm{~Hz}, 12 \mathrm{H}), 4.0$ । (doublet of septet, $J=3.7,2.3 \mathrm{~Hz}, 1 \mathrm{H}), 6.22(\mathrm{dd}, J=18.7,2.3 \mathrm{~Hz}, 1 \mathrm{H}), 6.96-6.98(\mathrm{~m}, 2 \mathrm{H}), 7.05(\mathrm{~d}, J=$ $18.7 \mathrm{~Hz}, 1 \mathrm{H}), 7.18$ (d, $J=4.6 \mathrm{~Hz}, 1 \mathrm{H}) ;{ }^{13} \mathrm{C} \mathrm{NMR}\left(\mathrm{CDCl}_{3}, 75 \mathrm{MHz}\right) \delta-4.1,126.4,126.6,137.9,144.3$ : MS $\mathrm{m} / \mathrm{z}$ (rel intensity) $168\left(\mathrm{M}^{+}, 48\right), 153(100), 141$ (1.0), $127(41), 75(1.0)$; HRMS Calcd for $\mathrm{C}_{12} \mathrm{H}_{20} \mathrm{Si}_{2} \mathrm{~S}$ : 252.0824; Found: 252.0826 .

2,2'-p-Phenylene-bis-5-[ $\beta$-(2-dimethylsilyl)vinyl]thiophene (5c). In a manner similar to that described in the general procedure, a mixture of $14 \mathrm{c}(1.32 \mathrm{~g}, 2.5 \mathrm{mmol})$ was treated with $\mathrm{LiAlH}_{4}(0.4 \mathrm{~g}$. $10.0 \mathrm{mmol})$ to give $5 \mathrm{c}(0.75 \mathrm{~g}, 73 \%)$ : $\mathrm{mp} 170-181^{\circ} \mathrm{C}$; ${ }^{1} \mathrm{H} \mathrm{NMR}\left(\mathrm{CDCl}_{3}, 200 \mathrm{MHz}\right) \delta 0.22(\mathrm{~d}, J=3.8 \mathrm{~Hz} .12$ H), 4.18 (doublet of septet, $J=3.8,2.7 \mathrm{~Hz}, 2 \mathrm{H}$ ), $6.19(\mathrm{dd}, J=18.9,2.7 \mathrm{~Hz}, 2 \mathrm{H}), 6.94(\mathrm{~d}, J=3.9 \mathrm{~Hz}, 2$ H), $7.00(\mathrm{~d}, J=18.9 \mathrm{~Hz}, 2 \mathrm{H}), 7.20(\mathrm{~d}, J=3.9 \mathrm{~Hz}, 2 \mathrm{H}), 7.57(\mathrm{~s}, 4 \mathrm{H}) ;{ }^{13} \mathrm{C} \mathrm{NMR}\left(\mathrm{CDCl}_{3}, 75 \mathrm{MHz}\right) \delta-4.0$, $123.5,126.0,126.1,127.2,133.4,137.8,143.1,144.4 ; \mathrm{MS} \mathrm{m} / \mathrm{z}$ (rel intensity) $410\left(\mathrm{M}^{+}, 100\right), 395$ (19), 352 (11), 337 (10), 320 (21), 305 (27), 59 (32); HRMS Calcd for $\mathrm{C}_{22} \mathrm{H}_{26} \mathrm{Si}_{2} \mathrm{~S}_{2}: 410.1014$; Found: 410.1019.

$5,5^{\prime \prime}$-Bis[ $\beta$-(2-dimethylsilyl)vinyl]-2,2';5'2"-terthiophene (5d). In a manner similar to that described in the general procedure, a mixture of $14 \mathrm{~d}(1.33 \mathrm{~g}, 2.5 \mathrm{mmol})$ was treated under $\mathrm{N}_{2}$ with $\mathrm{LiAlH}_{4}$ $(0.4 \mathrm{~g}, 10.0 \mathrm{mmol})$ to give $5 \mathrm{~d}(0.69 \mathrm{~g}, 67 \%): \mathrm{mp} 157-158{ }^{\circ} \mathrm{C} ;{ }^{1} \mathrm{H}$ NMR $\left(\mathrm{CDCl}_{3}, 200 \mathrm{MHz}\right) \delta 0.21(\mathrm{~d}, J=3.6$ $\mathrm{Hz}, 12 \mathrm{H}$ ), 4.16 (doublet of septet, $J=3.6,2.7 \mathrm{~Hz}, 2 \mathrm{H}$ ), $6.14(\mathrm{dd}, J=18.7,2.7 \mathrm{~Hz}, 2 \mathrm{H}), 6.86(\mathrm{~d}, J=3.7$ $\mathrm{Hz}, 2 \mathrm{H}), 6.96(\mathrm{~d}, J=18.7 \mathrm{~Hz}, 2 \mathrm{H}), 7.02(\mathrm{~d}, J=3.7 \mathrm{~Hz}, 2 \mathrm{H}), 7.05(\mathrm{~s}, 2 \mathrm{H}) ;{ }^{13} \mathrm{C} \mathrm{NMR}\left(\mathrm{CDCl}_{3}, 75 \mathrm{MHz}\right) \delta$ $-4.0,124.0,124.3,124.4,126.3,127.0,136.4,137.5,143.9 ; \mathrm{MS} \mathrm{m} / \mathrm{z}$ (rel intensity) $416\left(\mathrm{M}^{+}, 100\right), 326$ (21), 311 (10), 268 (22), 236 (81); HRMS Calcd for $\mathrm{C}_{20} \mathrm{H}_{24} \mathrm{Si}_{2} \mathrm{~S}_{3}$ : 416.0578 ; Found: 416.0576.

2,5-Bis $\left\{\beta\right.$-[dimethyl( $\beta^{\prime}$-styryl)silyl]vinyl $\}$ thiophene (15a). A mixture of $5 \mathrm{~b}(0.25 \mathrm{~g}, 1.0$ $\mathrm{mmol})$, phenylacetylene $(0.20 \mathrm{~g}, 2.0 \mathrm{mmol})$ and $\mathrm{RhCl}\left(\mathrm{PPh}_{3}\right)_{3}(4.6 \mathrm{mg}, 0.5 \mathrm{~mol} \%)$ in THF $(5 \mathrm{~mL})$ was stirred under $\mathrm{N}_{2}$ at $50^{\circ} \mathrm{C}$ for $4 \mathrm{~h}$. The solvent was removed in vacuo, and the residue was chromatographed on silica gel (hexane) to give the product $15 \mathrm{a}(0.36 \mathrm{~g}, 79 \%) ;{ }^{1} \mathrm{H} \mathrm{NMR}\left(\mathrm{CDCl}_{3}, 300 \mathrm{MHz}\right) \delta 0.29(\mathrm{~s}, 12 \mathrm{H}), 6.22(\mathrm{~d}, J$ $=18.7 \mathrm{~Hz}, 2 \mathrm{H}), 6.48(\mathrm{~d}, J=19.3 \mathrm{~Hz}, 2 \mathrm{H}), 6.83(\mathrm{~s}, 2 \mathrm{H}), 6.89-6.97(\mathrm{~m}, 4 \mathrm{H}), 7.22-7.34(\mathrm{~m}, 6 \mathrm{H}), 7.44$ $(\mathrm{m}, 4 \mathrm{H}) ;{ }^{13} \mathrm{C} \mathrm{NMR}\left(\mathrm{CDCl}_{3}, 75 \mathrm{MHz}\right) \delta-2.6,126.4,126.6,127.0,127.7,128.1,128.5,137.9,138.1$, 144.5, 145.0; MS m/z (rel intensity) $456\left(\mathrm{M}^{+}, 32\right), 365$ (14), 306 (12), 161 (40), 145 (65); 135 (42), 59 (100); HRMS Calcd for $\mathrm{C}_{28} \mathrm{H}_{32} \mathrm{Si}_{2} \mathrm{~S}: 456.1763$; Found: 456.1757 .

2,2'-p-Phenylene-bis-5-\{[ $\beta$-dimethyl $(\beta$-styryl)silyl]vinyl $\}$ thiophene (15b). In a manner similar to that described above, a mixture of $5 \mathrm{c}(0.21 \mathrm{~g}, 0.5 \mathrm{mmol})$, phenylacetylene $(0.10 \mathrm{~g}, 1.0 \mathrm{mmol})$ and $\mathrm{RhCl}\left(\mathrm{PPh}_{3}\right)_{3}(2.3 \mathrm{mg}, 0.5 \mathrm{~mol} \%)$ in THF $(5 \mathrm{~mL})$ was transformed into $15 \mathrm{~b}(0.24 \mathrm{~g}, 78 \%):{ }^{1} \mathrm{H} \mathrm{NMR}$ $\left(\mathrm{CDCl}_{3}, 300 \mathrm{MHz}\right) \delta 0.32(\mathrm{~s}, 12 \mathrm{H}), 6.28(\mathrm{~d}, J=18.8 \mathrm{~Hz}, 2 \mathrm{H}), 6.52(\mathrm{~d}, J=19.0 \mathrm{~Hz}, 2 \mathrm{H}), 6.97(\mathrm{~d}, J=$ $19.0 \mathrm{~Hz}, 2 \mathrm{H}), 6.96(\mathrm{~d}, J=3.7 \mathrm{~Hz}, 2 \mathrm{H}), 7.00(\mathrm{~d}, J=18.8 \mathrm{~Hz}, 2 \mathrm{H}), 7.21(\mathrm{~d}, J=3.7 \mathrm{~Hz}, 2 \mathrm{H}), 7.26-7.37$ $(\mathrm{m}, 6 \mathrm{H}), 7.47(\mathrm{~d}, J=7.8 \mathrm{~Hz}, 4 \mathrm{H}), 7.59(\mathrm{~s}, 4 \mathrm{H}) ;{ }^{13} \mathrm{C} \mathrm{NMR}\left(\mathrm{CDCl}_{3}, 50 \mathrm{MHz}\right) \delta-2.6,123.5,125.9,126.5$, $127.0,127.2,127.4,128.1,128.4,128.5,137.4,138.1,143.0,144.6,145.1 ; \mathrm{MS} \mathrm{m} / \mathrm{z}$ (rel intensity) 614 $\left(M^{+}, 86\right), 441(23), 211(12), 161(11), 145(100), 135$ (34), 73 (28), 59 (73); HRMS Calcd for $\mathrm{C}_{38} \mathrm{H}_{38} \mathrm{Si}_{2} \mathrm{~S}_{2}$ : 614.1953; Found: 614.1958 . 
1,4-Bis $\left\{\beta\right.$-\{dimethyl[ $\beta^{\prime}$-(2-thienyl)vinyl]silyl\}vinyl\}benzene (15c). In a manner similar to that described above, a mixture of $12 \mathrm{~b}(0.67 \mathrm{~g}, 4.0 \mathrm{mmol}), 1,4$-diethynylbenzene $(0.25 \mathrm{~g}, 2.0 \mathrm{mmol})$ and $\mathrm{RhCl}\left(\mathrm{PPh}_{3}\right)_{3}$ (18.5 mg, $\left.0.5 \mathrm{~mol} \%\right)$ in THF $(20 \mathrm{~mL})$ was converted to $15 \mathrm{c}(0.74 \mathrm{~g}, 80 \%):{ }^{1} \mathrm{H} \mathrm{NMR}_{(\mathrm{CDCl}}$, $300 \mathrm{MHz}) \delta 0.28(\mathrm{~s}, 12 \mathrm{H}), 6.23(\mathrm{~d}, J=18.9 \mathrm{~Hz}, 2 \mathrm{H}), 6.48(\mathrm{~d}, J=19.1 \mathrm{~Hz}, 2 \mathrm{H}), 6.91(\mathrm{~d}, J=19.1 \mathrm{~Hz}, 2$ H), 6.95-7.03 (m, $6 \mathrm{H}), 7.17$ (br d, $J=4.7 \mathrm{~Hz}, 2 \mathrm{H}), 7.41(\mathrm{~s}, 4 \mathrm{H}) ;{ }^{3} \mathrm{C} \mathrm{NMR}\left(\mathrm{CDCl}_{3}, 75 \mathrm{MHz}\right) \delta-2.6$, $124.9,125.9,126.4,126.7,127.0,127.3,127.5,137.4,138.0,144.5 ; \mathrm{MS} \mathrm{m} / \mathrm{z}$ (rel intensity) $462\left(\mathrm{M}^{+}, 5\right.$ ), 365 (14), 350 (4), 283 (20), 242 (19), 199 (28), 167 (68), 141 (100), 73 (55), 59 (53); HRMS Calcd for $\mathrm{C}_{26} \mathrm{H}_{30} \mathrm{Si}_{2} \mathrm{~S}_{2}: 462.1327$; Found: 462.1327.

Poly(1,4-divinylbenzene-dimethylsilylene-2,5-divinylthiophene-dimethylsilylene) (6a). A mixture of $5 \mathrm{~b}(0.25 \mathrm{~g}, 1.0 \mathrm{mmol}), 1,4$-diethynylbenzene $(0.13 \mathrm{~g}, 1.0 \mathrm{mmol})$ and $\mathrm{RhCl}\left(\mathrm{PPh}_{3}\right)_{3}(4.6 \mathrm{mg}, 0.5$ $\mathrm{mol} \%)$ in THF ( $5 \mathrm{~mL}$ ) was stirred at $50^{\circ} \mathrm{C}$ for $4 \mathrm{~h}$. After cooling, methanol was added to precipitate the crude polymer. After filtration, the solid was washed twice with methanol and dried to give $6 \mathrm{a}(0.32 \mathrm{~g}, 87 \%) ; \mathrm{M}_{\mathrm{n}}=$ 5535 (polydispersity 2.4); ${ }^{1} \mathrm{H} \mathrm{NMR}\left(\mathrm{CDCl}_{3}, 300 \mathrm{MHz}\right) \delta 0.27$ (br s, $\left.12 \mathrm{H}\right), 6.21(\mathrm{br} \mathrm{d}, J=18.7 \mathrm{~Hz}, 2 \mathrm{H}$ ), 6.47 (br d, $J=19.2 \mathrm{~Hz}, 2 \mathrm{H}$ ), $6.82(\mathrm{br}, 2 \mathrm{H}$ ), 6.90 (br d, $J=19.2 \mathrm{~Hz}, 2 \mathrm{H}$ ), $6.92(\mathrm{br} \mathrm{d}, J=18.7 \mathrm{~Hz}, 2 \mathrm{H}$ ), $7.40(\mathrm{br}, 4 \mathrm{H})$.

Poly(1,4-divinylbenzene-dimethylsilylene-2,2'-p-phenylene-bis-(5-vinylthiophene)-dimethylsilylene (6b). In a manner similar to that described above, a mixture of $5 \mathbf{c}(0.21 \mathrm{~g}, 0.5 \mathrm{mmol}), 1,4-$ diethynylbenzene $(63 \mathrm{mg}, 0.5 \mathrm{mmol})$ and $\mathrm{RhCl}\left(\mathrm{PPh}_{3}\right)_{3}(2.3 \mathrm{mg}, 0.5 \mathrm{~mol} \%)$ in THF (2.5 ml) was transformed into $6 \mathrm{~b}(0.21 \mathrm{~g}, 80 \%): \mathrm{M}_{\mathrm{n}}=6167$ (polydispersity 2.0); ${ }^{1} \mathrm{H} \mathrm{NMR}\left(\mathrm{CDCl}_{3}, 300 \mathrm{MHz}\right) \delta 0.31$ (br s, $12 \mathrm{H}$ ), 6.26 (br d, $J=18.2 \mathrm{~Hz}, 2 \mathrm{H}$ ), 6.51 (br d, $J=19.2 \mathrm{~Hz}, 2 \mathrm{H}$ ), 6.59 (br, $6 \mathrm{H}$ ), 7.20-7.24 (br, $4 \mathrm{H}$ ), 7.43- 7.58 (br, 8 H).

Acknowledgments. The authors wish to thank the National Science Council of the Republic of China for financial support.

\section{References}

1. (a) Hu, S. S.; Weber, W. P. Weber, Polym. Bull.1989, 2l, 133. (b) Ishikawa, M.; Ni, H.; Watanabe, H.; Saheki, Y.Organometallics 1987, 6. 1673. (c) Ohshita, J.; Matsuguchi, A.; Furumori, K.; Hong, R.F.: Ishikawa, M. Macromolecules 1992, 25, 2134. (d) Ohshita, J.; Kanaya, D.; Watanabe, T.; Ishikawa, M. J. Organomet. Chem. 1995, 489, 165.

2. (a) Ohshita, J.; Kanaya, D.; Ishikawa, M.; Koike: T.; Yamanaka, T.Macromolecules 1991, 24, 2106. (b) Corriu, R. J. P.; Guerin, C.; Henner, B.; Kuhlmann, T.; Jean, A.; Garnier, F.; Yassar, A. Chem. Mater. 1990, 2, 351. (c) Ohshita, J.; Kanaya, D.; Ishikawa, M. J. Organomet. Chem. 1989, 369, C18. (d) Yuan, C.-H.; West, R. Appl. Organomet. Chem 1994, 8, 423.

3. Corriu, R. J. P.; Douglas, W. E.; Yang, Z. X.; Karakus, Y.; Cross, G. H.; Bloor, D.J. Organomet. Chem. 1993, 455, 69.

4. (a) Burroughes, J. H.; Gradley, D. D. C.; Brown, A. R.; Marks, R. N.; Mackay, K.; Friend, R. H.: Burn, P. L.; Holmes, A. B. Nature 1990, 347, 539. (b) Greenham, N. C.; Moratti, S. C.; Bradley, D. D. C.; Friend, R. H.; Holmes, A. B. Nature 1993, 365. 628. (c) Halls, J. J. M.; Walsh, C. A.; Greenham. N. 
C.; Marseglia, E. A.; Friend, R. H.; Moratti, S. C.; Holmes, A. B. Nature 1995, 376, 498.

5. (a) Egelhaaf, H.-J.; Oelkrug, J. G. D. Synth. Met. 1996, 83, 221. (b) Oelkrg, D.; Egelhaaf, H.-J.; Gierschner, J.; Tompert, A. Synth. Met. 1996, 76, 249. (c) Maddux, T.; Li, W.; Yu, L. J. Am. Soc. Chem. 1997, 1/9, 844.

6. (a) Ryu, M.-K; Lee, S.-M.; Zyung, T.; Kim, H. K. Polym. Mater. Sci. Eng. 1996, 75 (2), 408. (b) Adachi, C.; Tautsui, T.; Saito, S. Appl. Phys. Lett. 1990, 56, 799. (c) Pope, M.; Swenberg, C. E. Electronic Processes in Organic Crystals; Oxford U: Oxford, 1982. (d) Kido, J.; Kohda, M.; Okuyama, K.; Nagai, K. Appl. Phys. Lett. 1992, 61, 761. (e) Yang, Z.; Sokolik, I.; Karasz, F. E. Macromolecules 1993, 26, 1188. (f) Kim, D. J.; Kim. S. H.; Lee, J. H.; Kang, S. J.; Kim, H. K.; Zyung, T.; Cho, I.; Choi, S. K. Mol. Cryst. Liq. Cryst. 1996, 280, 391.

7. Kim, H. K.; Ryu, M.-K.; Lee, S.-M. Macromolecules 1997, 30, 1236.

8. For a recent review on hydrosilylation, see: Ojima, I. In The Chemistry of Organosilicon Compounds; Vol. 2, Rappoport, Z.; Apeloig, Y. Eds.; Wiley: Chicester, 1997; in press.

9. Pang, Y.; Ijadi-Maghsoodi, S.; Barton, T, J. Macromolecules 1993, 26, 5671

10. (a) Son, D, Y.; Bucca, D.; Keller, T. M. Tetrahedron Lett. 1996, 37, 1579. (b) Kunai, A.; Toyoda, E.; Nagamoto, I.; Horio, T.; Ishikawa, M. Organometallics 1996, $15,75$.

11. Chen, R.-M.; Chien, K.-M.; Wong, K.-T.; Jin, B.-W.; Luh, T.-Y.; Hsu, J.-L.; Fann, W. manuscript in preparation.

12. (a) Chan, T.-H.; Chen, L. M.; Wang, D. J. Chem. Soc., Chem. Co mmun. 1988, 1280. (b) White, D. G.; Rochow, E. G. J. Am. Chem. Soc. 1954, 76, 3897. (c) Skell, P. S.; Goldstein, E. J. J. Am. Chem. Soc. 1964, 86, 1442. (d) Curry, J. W. J. Am. Chem. Soc. 1956, 78, 1686.

13. For reviews, see: (a) Luh, T.-Y. Acc. Chem. Res. 1991, 24, 257. (b) Luh, T.-Y.; Wong, K.-T. Synthesis 1993, 349. (c) Luh, T.-Y.; Leung, M.-K. In Advances in the Use of Synthons in Organic Chemistry; Dondoni, A., Ed.; JAI: London, Vol 2, 1995, 129. (d) Luh, T.-Y. Pure Appl. Chem. 1996, 68, 105.

14. (a) Ni, Z.-J.; Luh, T.-Y. J. Org. Chem. 1988, 53, 2129, 5582. (b) Ni, Z.-J.; Yang, P.-F.; Ng, D. K. P.; Tzeng, Y.-L.; Luh, T.-Y. J. Am. Chem. Soc. 1990, 112, 9356. (c) Wong, K.-T.; Yuan, T.-M.; Wang, M. C.; Tung, H.-H.; Luh, T.-Y. J. Am. Chem. Soc. 1994, 116, 8920. (d) Ni, Z.-J.; Luh, T.-Y. Org. Syn. 1991, 70, 240.

15. (a) Schmidbaur, H. Waldmann, S. Chem. Ber. 1964, 97, 3381. (b) Jarvie, A. W. P.; Rowley, R. J. J. Organomet. Chem. 1972, 34, C7.

16. (a) Noll, W. Chemistry and Technology of Silicones; Academic Press: New York, 1968; p 87. (b) Wilberg, N. In Progress in Organosilicon Chemistry; Marciniec, B.; Chojnowski, J., Eds.; Gordon \& Breach: Basel, 1995; p. 19.

17. Tretner, C.; Zobel, B.; Hummeltenberg, R.; Uhlig, W. J. Organomet. Chem. 1994, 468, 63.

18. Ohshita, J.; Yamashita, A.; Hiraoka, T.; Shinpo, A.; Kunai, A.; Ishikawa, M. Macromolecules 1997, 30 , 1540.

19. Tamao, K.; Ishida, N.; Ito, Y.; Kumada, M. Org. Syn. 1990, 69, 96. 